\title{
Chapter 6 \\ How to Design Quality Light Sources With Discrete Color Components
}

\begin{abstract}
White light sources using discrete emitters require careful design and optimization. The first step of the design should be determining the intended use of the light source so that application specific requirements can be addressed. Subsequently, optimal designs made of discrete emitters should be determined and finally, experimental implementation of the light source should be carried out. In this Chapter of the brief, we limit ourselves to the use of discrete emitters for indoor and outdoor lighting together with display backlighting applications. For each application, we summarize the requirements that need to be satisfied and present design guidelines to implement quality light sources made of discrete emitters.
\end{abstract}

Keywords Optimization of photometric quantities • Indoor lighting • Outdoor lighting $\cdot$ Display backlighting

\subsection{Advanced Design Requirements for Indoor Lighting}

For indoor lighting applications it is essential for a light source to reflect the real colors of objects. This requires CRI and/or CQS to be maximized. Furthermore, the light source needs to have a warm white shade for a comfortable vision, necessitating CCTs lower than $4500 \mathrm{~K}$ (preferably $<4000 \mathrm{~K}$ ). Also, the overlap with the human eye sensitivity function should be maximized, which is quantified by LER. To ensure energy saving, LE of the device should be maximized as well. Ideally, for LER we expect to reach values greater than $350 \mathrm{~lm} / \mathrm{W}_{\text {opt }}$ while for the LE targeted levels should be $>100-120 \mathrm{~lm} / \mathrm{W}_{\text {elect }}$ to compete with the existing light sources. In this part of the brief, we summarize the design strategies in the light of Refs. [1] and [2] to optimize all the parameters for a high-quality light-emitting device. We start with the necessary conditions to realize high-quality white light spectrum for indoor lighting and then continue with summarizing the requirements for high device efficiency.

Optimizing the spectral features of white light sources employing discrete emitters is a complicated task. To have a qualitative picture while approaching this problem, one needs to have an idea on the trade-offs between several performance metrics of interest. In Ref. [1], this problem was addressed by calculating the performance of 
Fig. 6.1 CRI versus LER trade-off for nanocrystal-integrated white LEDs at different CCTs. Reproduced with permission from Ref. [4] () Science Wise Publishing \& DeGruyter 2013

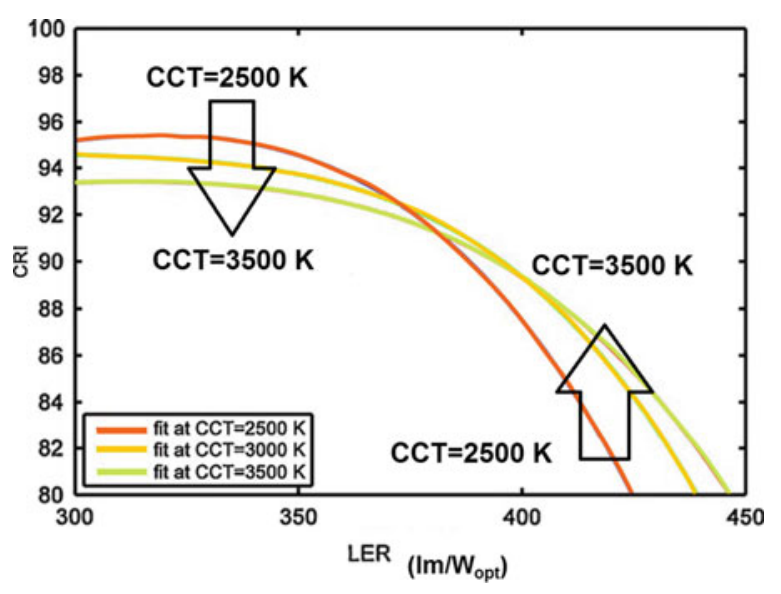

modelled white LED spectra made of colloidal nanocrystal quantum dots using realistic properties. The first point that needs to be clarified is the number of color components. It turns out that when narrow emitters such as quantum dots are employed, utilizing only three color components (i.e., blue, green, and red) cannot provide sufficient degrees of freedom to optimize photometric properties and achieve high-quality lighting. As explained in Ref. [1] and also stated by Tsao [3], the minimum number of color components needed is four which can be blue, green, yellow, and red. For a good white light source, a good white light source should be able to render the real colors of the objects. When narrow emitters such as colloidal quantum dots or individual LED chips that have emission linewidths between $20-50 \mathrm{~nm}$ are employed and when only three colors or less are used, the spectrum of the light source cannot sufficiently span the whole visible regime leading to poor color rendition performance which translates to low CRI or CQS values. Nevertheless, it turns out that employing four color components is enough to solve this problem and achieving good color rendition is possible while also optimizing other parameters.

The analyses presented in Ref. [1] also show that there is a fundamental trade-off between LER and CRI. At a fixed CCT value, as a general trend, the increase of LER is accompanied by a decrease in the CRI and vice versa (Fig. 6.1). An interesting finding is that the maximum CRI values that can be obtained at a given LER favour warmer white lights until $\sim 370 \mathrm{~lm} / \mathrm{W}_{\text {opt }}$ whereas after this value high CRI values can be obtained at the expense of cooler white shades. All in all, below $\sim 370 \mathrm{~lm} / \mathrm{W}_{\text {opt }}$, CRIs $>92$ are feasible. However, beyond $\sim 370 \mathrm{~lm} / \mathrm{W}_{\text {opt }}$, it is not possible to sustain CRI levels above 90.

The emission wavelength, relative amplitude, and linewidth of each color component has a profound effect on the performance of the designed white light source. The results obtained from simulations are presented in Fig. 6.2. As seen in this figure, high-quality lighting depends strongly on the properties of the red color component. The peak emission wavelength of this color component should be at $620 \mathrm{~nm}$ in photopic regime. The designer does not have a large flexibility to play with this 


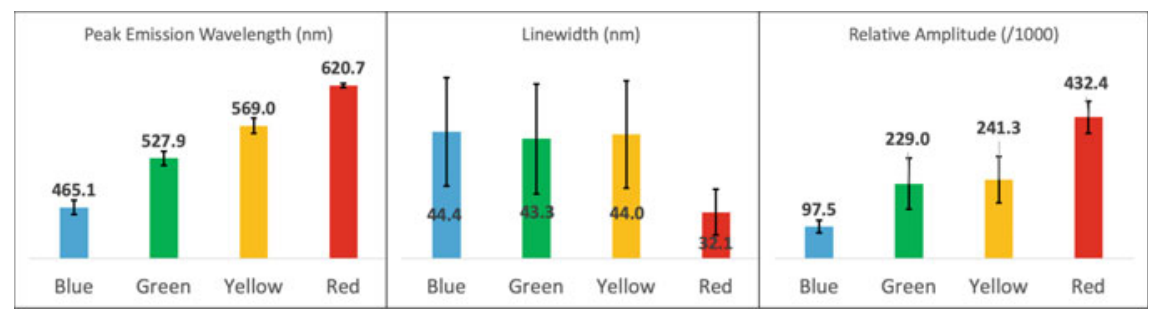

Fig. 6.2 Average and standard deviations of the peak emission wavelength, linewidth and relative amplitude of nanocrystal quantum dot color components to obtain white LED spectra possessing CRI $>90$, LER $>380 \mathrm{~lm} / \mathrm{W}_{\text {opt }}$, and CCT $<4000 \mathrm{~K}$

value due to the low standard deviation shown in Fig. 6.2. Furthermore, this color component should be the most dominant one in the white light spectrum. Second important condition on high-quality lighting is imposed by the blue component. Due to warm white light requirement, the amplitude of the blue color component has to be strictly placed around 90/1000. As in the case of red color component, the designer does not again have the flexibility to change this value without sacrificing from the overall performance. On the other hand, the computations indicate there is no major restriction on the linewidth of this color component. Furthermore, the peak emission wavelength can be flexibly chosen around $465 \mathrm{~nm}$ as the large standard deviations in Fig. 6.2 imply. It turns out that the designer has the highest flexibility when choosing the green and yellow color components compared to the blue and the red. The simulations show that the peak emission wavelengths of these colors can be selected around 528 and $569 \mathrm{~nm}$ without a strong restriction as the corresponding large standard deviations indicate. Furthermore, both broad and narrow emission spectra of green and yellow components are found to allow for high performance. Finally, the relative amplitudes of 229/1000 and 241/1000 with standard deviations > 70/1000 for green and yellow components, respectively, mean that the amplitudes of these colors should be between the amplitudes of the blue and red color components. It turns out that the designer has a large flexibility to play with these parameters without sacrificing the end performance of the device. The white LED spectrum generated using these average values possesses a CRI of 91.3, an LER of $386 \mathrm{~lm} / \mathrm{W}_{\text {opt }}$, and a CCT of $3041 \mathrm{~K}$ (Fig. 6.3). This shows that extremely high-quality artificial white light can be generated via color conversion by employing nanocrystal quantum dots integrated with LEDs as optical pumps when the requirements listed above are addressed.

After establishing the requirements for high-quality white light spectrum generation, the next essential question of LED design is how to place the nanocrystal quantum dots on top of the blue LED, and what the performance limits in terms of energy consumption are. In Ref. [2], the answers to these questions are sought through a computational approach. In this work, the potential luminous efficiency of LEDs deploying two types of nanocrystal quantum dot color converting thin film architectures are investigated. These are (i) three separate layers of nanocrystal quan- 
Fig. 6.3 Designed spectrum of nanocrystal quantum dot integrated white LED generated using the results in Fig. 6.2 along with a summary of its performance in the inset. Reproduced with permission from Ref. [4]

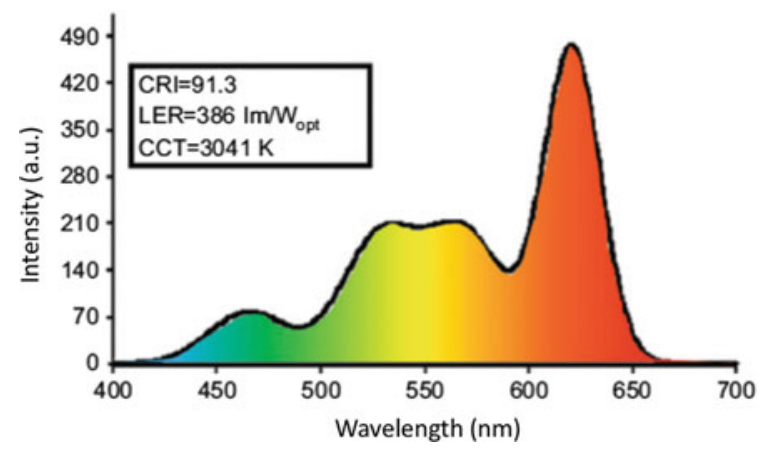

tum dots (with the green first, followed by the yellow, and then the red on top) and (ii) the nanocrystal blended together to form a single coating layer on a blue LED.

To investigate the ultimate limits, the hypothetical case of quantum dot films having unity quantum efficiency is simulated. It turns out that the energy loss due to photon energy down-conversion is at least $17 \%$ when a photometrically efficient design is employed. This corresponds to a luminous efficiency of $315 \mathrm{~lm} / \mathrm{W}_{\text {elect }}$ when a perfect blue LED chip with a power conversion efficiency of $100 \%$ is assumed. This means a highly efficient blue LED having a power conversion efficiency of $81.3 \%$ is used [5], the maximum obtainable luminous efficiency becomes $256 \mathrm{~lm} / \mathrm{W}_{\text {elect }}$. When a typical blue LED efficiency of $40 \%$ is considered, then the maximum obtainable LE becomes $126 \mathrm{~lm} / \mathrm{W}_{\text {elect }}$. This shows that one of the most critical conditions to achieve a highly efficient design is to realize highly efficient blue LED chips.

Another important question is how to construct the nanocrystal thin films to realize high efficiency. The results of Ref. [2] show that the layered architecture outperforms the blended architecture in terms of overall luminous efficiency. In order to achieve high photometric performance together with a luminous efficiency level of 100,150 , and $200 \mathrm{~lm} / \mathrm{W}_{\text {elect }}$, nanocrystals quantum dots need to have quantum efficiency of at least 43,61 , and $80 \%$, respectively, if an LED with a power conversion efficiency of $81.3 \%$ is employed. On the other hand, when utilizing the blended quantum dot white LED architecture, the required quantum efficiencies of the QDs need to increase to 47,65 , and $82 \%$, respectively. This is essentially what one should expect. In a layered architecture where the green nanocrystals are on top and the red nanocrystals are at the bottom, the energy loss due to color conversion is minimized compared to the blend architecture. If the order of the layered nanocrystals is reversed, the resulting efficiency remains below that of the blend film due to increased radiative energy transfer from nanocrystals emitting high energy photons to nanocrystals emitting low energy photons. 


\subsection{Advanced Design Requirements for Outdoor Lighting}

Different than indoor lighting, for outdoor lighting the important point is to achieve a lighting condition that can help the pedestrians and drivers recognize their surroundings. For that purpose, the perceived luminance plays a critical role while the shade of the white and color rendition performance are not as critical as in the case of indoor lighting. Nevertheless, in the literature a light source having a color rendering index $>80$ was found to increase the visual acuity under street lighting conditions. The critical difference to consider here is the change in the eye sensitivity function under the mesopic visual regime conditions where the rods and cones contribute to the vision together.

To take this variation into account, in Ref. [6] the design requirements for achieving high-quality lighting with nanocrystal quantum dots are established. This study starts with determining the luminance levels to satisfy the existing road lighting standards, which vary significantly depending on the country. For this purpose, four different luminance levels were selected such that the British [7] and US [8] standards can be fulfilled. These are $0.50,0.80,1.25$, and $1.75 \mathrm{~cd} / \mathrm{m}^{2}$ which are named in Ref. [6] as Mesopic 1, 2, 3, and 4, respectively. Next, the performance of the commercial white light sources was evaluated. Among a cool white fluorescent lamp (CWFL), an incandescent lamp with a CCT of $3000 \mathrm{~K}$, a metal-halide lamp (MH), a high-pressure sodium lamp (HPS), and a mercury vapor lamp (MV), the CWFL was found the most efficient commercial light source for Mesopic 1 and 2 standards, whereas the HPS becomes the most efficient source for Mesopic 3 and 4 standards. To test the potential of nanocrystal quantum dot integrated white LEDs, spectra having the same radiance as the CWFL has for Mesopic 1 and 2 standards and HPS has for Mesopic 3 and 4 are generated. Among ca. 100 million spectra tested, the ones possessing CRI or CQS > 85 and higher luminance than the commercial light source are investigated. Results indicate that using nanocrystal quantum dots, it is possible to simultaneously obtain high mesopic luminance as well as successful color rendition performance and warm white appearance. Having a blue component close to $460 \mathrm{~nm}$ and a red component around $610 \mathrm{~nm}$ turn out to be crucial for realizing this performance (Fig. 6.4). In addition, the weight of the blue component should be around 150/1000, whereas the relative intensity of the red component should be chosen around 450/1000. Different than the indoor lighting conditions, the outdoor lighting requirements do not pose strong limitations on the emission linewidths of each color component. Results summarized in Fig. 6.4 show that bandwidths of 35-40 $\mathrm{nm}$ can lead to the generation of high-quality road lighting.

Maintaining high electrical efficiency is another important concern while designing a white light source. To answer what efficiency levels can make the nanocrystal quantum dot integrated LEDs more efficient than the commercial light sources, a systematic calculation has been carried out in Ref. [6]. Assuming that the power conversion efficiencies of CWFL as $28 \%$, HPS as $31 \%$, and MH as $24 \%$, the quantum dot integrated LEDs satisfying high quality road lighting conditions can make 
(a)

(b)
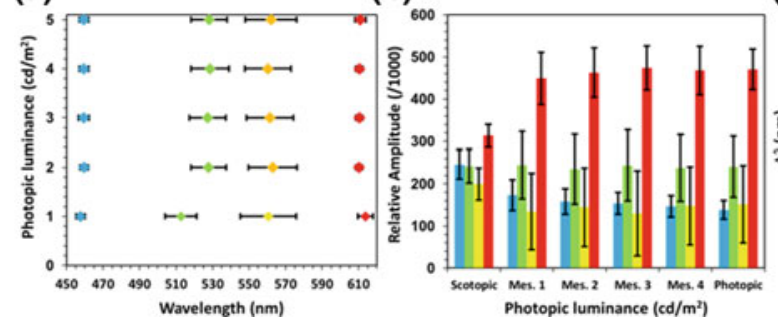

(c)

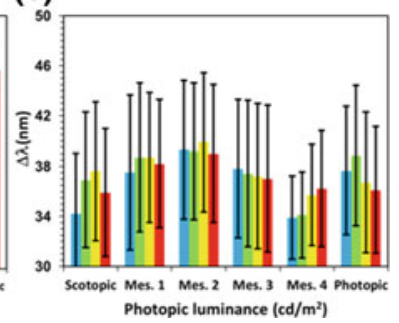

Fig. 6.4 Average and standard deviation (bars) of (a) peak emission wavelength, relative amplitude, and (c) full-width at half maximum belonging to the spectra suitable for road lighting conditions. Colors in the graph indicate the corresponding color components. Reproduced with permission from Ref. [6] (C) Science Wise Publishing \& DeGruyter 2014

more efficient street lighting sources if the final power conversion efficiency of the device is larger than $24 \%$, which was also shown to be experimentally feasible [6].

\subsection{Advanced Design Requirements for Display Backlighting}

Together with the energy consumption, the ability to reproduce the real colours of objects is one of the most desired properties for a liquid crystal display. These displays rely on a backlight which is a white light source that spans three colours, i.e., blue, green, and red. This white light passes through polarizers and then liquid crystals of each pixel that let the light pass through or block it. Next, the light reaches the blue, green, or red color filters that produce the desired color. The color span (also referred to as color gamut) of a display is governed by the purity of each blue, green, and red color components as a display can only produce colors made of combinations of individual color components. Thus, the purer individual color components are, the wider the color span is. Therefore, the narrower each color component is, the wider the color gamut of the display becomes. The narrow color components can be obtained by utilizing color filters having very narrow transmission spectra. Nevertheless, this is not a good idea unless the individual color components are narrow enough because a significant portion of the energy would otherwise be wasted. Therefore, the correct choice here is utilizing narrow-band emitters, e.g., nanocrystal quantum dots, colloidal quantum wells, perovskite nanocrystals, and individual LED chips.

To have an idea on the potential of narrow emitters for displays, in Fig. 6.5 we show the color gamut of the National Television System Committee (NTSC) standard (dashed triangle) together with the potential color gamut of displays using quantum dot integrated backlight sources [4]. The black lines in the graph indicate the chromaticity coordinates of the quantum dots with peak emission wavelengths 


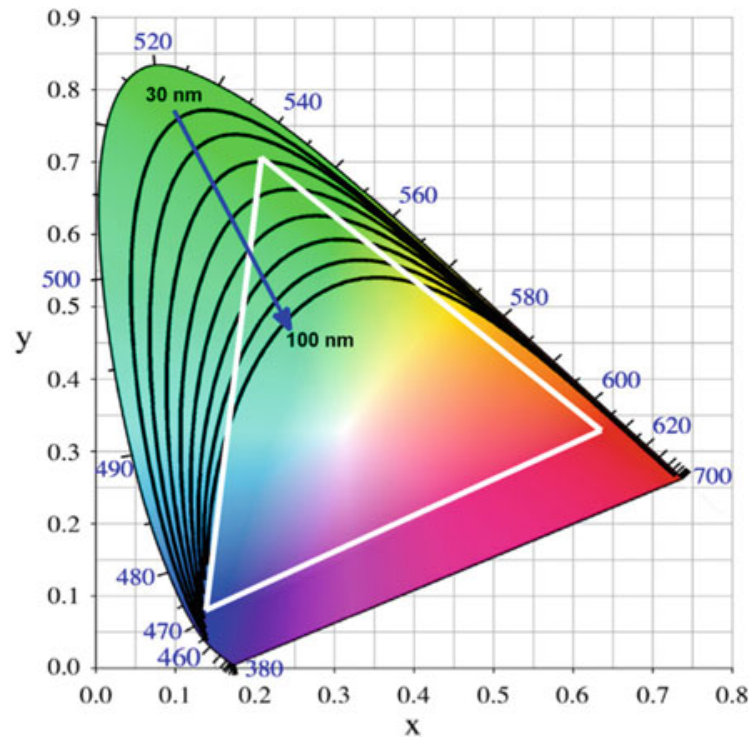

Fig. 6.5 NTSC color gamut in (x, y) color space (white triangle). The black lines indicate the colors that can be obtained using the nanocrystal quantum dots with different linewidths ranging from 30 to $100 \mathrm{~nm}$. Reproduced from Ref. [4] @ Science Wise Publishing \& DeGruyter 2013

varying from 460 to $700 \mathrm{~nm}$ with linewidths between 30 and $100 \mathrm{~nm}$. It is noticeable that carefully designed quantum dot backlight is able to produce a larger color gamut than the NTSC color gamut. This figure also tells us that the quantum dots should have a linewidth of $50 \mathrm{~nm}$ or narrower in order to satisfy the standard color gamut.

More detailed analyses on the requirements for a high-performance display employing nanocrystal quantum dots were carried by Luo et al. [9]. In this work, the performance of blue LED pumped color enrichment structure made of nanocrystal quantum dots was studied. The authors presented six different display backlighting designs that can achieve similar brightness values to those of the commercially available display technologies. These results summarized in Table 6.1 present designs for color gamuts of the designed displays in comparison with NTSC color gamut in (x, y) and $\left(u^{\prime}, v^{\prime}\right)$ color spaces. Here, we see that if correctly designed, quantum dot based display backlighting can achieve 120 and 140\% of the NTSC color gamut according to $(\mathrm{x}, \mathrm{y})$ and $\left(\mathrm{u}^{\prime}, \mathrm{v}^{\prime}\right)$ color spaces, respectively. To achieve a broad color gamut, the emission peaks of blue and red color components have to be around 445 and $635 \mathrm{~nm}$, respectively. The emission peak of the green color component seems to dictate the observed performance differences between two different color spaces. Another interesting feature is that the emission linewidth is not a strict requirement for the blue component, whereas narrow emitters definitely seem to be favored for the red and green color components.

At this point, it is worth commenting on the choice of color spaces for optimizing the display designs. If one wants to increase the number of colors that a display 
Table 6.1 Optimal designs for nanocrystal quantum dot integrated displays based on (x, y) and $\left(\mathrm{u}^{\prime}, \mathrm{v}^{\prime}\right)$ color spaces [10]

\begin{tabular}{|c|c|c|c|c|c|c|}
\hline & Design \#1 & Design \#2 & Design \#3 & Design \#4 & Design \#5 & Design \#6 \\
\hline$\lambda_{\text {blue }}(\mathrm{nm})$ & 454.1 & 452.0 & 450.1 & 449.7 & 447.6 & 443.2 \\
\hline$\lambda_{\text {green }}(\mathrm{nm})$ & 548.0 & 542.7 & 535.6 & 529.8 & 523.5 & 547.1 \\
\hline$\lambda_{\text {red }}(\mathrm{nm})$ & 606.5 & 611.3 & 615.0 & 621.8 & 634.8 & 635.7 \\
\hline$\Delta \lambda_{\text {blue }}(\mathrm{nm})$ & 20.1 & 20.6 & 20.1 & 20.9 & 20.0 & 20.0 \\
\hline$\Delta \lambda_{\text {green }}(\mathrm{nm})$ & 30.1 & 30.8 & 30.4 & 30.4 & 30.0 & 30.0 \\
\hline$\Delta \lambda_{\text {red }}(\mathrm{nm})$ & 30.5 & 30.3 & 30.3 & 30.6 & 30.0 & 30.0 \\
\hline$\alpha_{\text {blue }}(\%)$ & 48.2 & 47.1 & 46.1 & 42.8 & 37.3 & 44.8 \\
\hline$\alpha_{\text {green }}(\%)$ & 30.8 & 29.8 & 28.8 & 29.3 & 27.8 & 30.2 \\
\hline$\alpha_{\text {red }}(\%)$ & 20.9 & 23.1 & 25.1 & 27.9 & 34.9 & 24.9 \\
\hline $\begin{array}{l}\text { Color } \\
\text { Gamut/NTSC } \\
\text { Gamut (x, y) } \\
\text { color space) }\end{array}$ & 0.80 & 0.90 & 1.00 & 1.10 & 1.20 & 1.01 \\
\hline $\begin{array}{l}\text { Color } \\
\text { Gamut/NTSC } \\
\text { Gamut }\left(\mathrm{u}^{\prime}, \mathrm{v}^{\prime}\right) \\
\text { color space) }\end{array}$ & 1.00 & 1.09 & 1.16 & 1.23 & 1.31 & 1.40 \\
\hline
\end{tabular}

$\lambda$ stands for the peak emission wavelength

$\Delta \lambda$ is the full-width at half-maximum, and

$\alpha$ is the relative amplitude of a particular color component

can show, obviously the color gamut has to be broadened. Nevertheless, to make a fair comparison but more importantly in order to have more meaningful and correct results, a uniform color gamut has to be used. This necessity becomes obvious when we take a look at the CIE 1931 (x, y) color space. In this system, the green colors [sparsely spaced on $(\mathrm{x}, \mathrm{y})$ ] span a huge area whereas the blue and especially red colors are confined to smaller regions. An optimized design based on this system, therefore, will naturally focus on narrow green emitters and mostly disregard the spectral characteristics of blue and red emitters. On the other hand, the effects of each color components seem to be more balanced in uniform color spaces. However, in this case, we strongly urge the designers to employ modern uniform color spaces such as $\mathrm{L}^{*} \mathrm{a}^{*} \mathrm{~b}^{*}$ rather than old ones such as $\left(\mathrm{u}^{\prime}, \mathrm{v}^{\prime}\right)$. As it is usually the case in color science, the industry is hardly adapted to these facts related to colorimetry and still tends to utilize old systems in their designs. 


\section{References}

1. Erdem T, Nizamoglu S, Sun XW, Demir HV (2010) A photometric investigation of ultra-efficient LEDs with high color rendering index and high luminous efficacy employing nanocrystal quantum dot luminophores. Opt Express 18(1):340-347

2. Erdem T, Nizamoglu S, Demir HV (2012) Computational study of power conversion and luminous efficiency performance for semiconductor quantum dot nanophosphors on light-emitting diodes. Opt Express 20(3):3275-3295

3. Phillips JM et al (2007) Research challenges to ultra-efficient inorganic solid-state lighting. Laser Photonics Rev 1(4):307

4. Erdem T, Demir HV (2013) Color science of nanocrystal quantum dots for lighting and displays. Nanophotonics 2(1):57-81

5. Narukawa Y, Ichikawa M, Sanga D, Sano M, Mukai T (2010) White light emitting diodes with super-high luminous efficacy. J Phys D Appl Phys 43(35):354002

6. Erdem T, Kelestemur Y, Soran-Erdem Z, Ji Y, Demir HV (2014) Energy-saving quality road lighting with colloidal quantum dot nanophosphors. Nanophotonics 3(6):373-381

7. "British Standard BS 5489-1:2003 - Code of practice for the design of road lighting," 2003

8. "American National Standard for electric lamps-Specifications for the chromaticity of solid state lighting products." American National Standards Institute, 2011

9. Luo Z, Chen Y, Wu S-T (2013) Wide color gamut LCD with a quantum dot backlight. Opt Express 21(22):26269-26284 